Draft version November 17, 2021

Preprint typeset using $\mathrm{LAT}_{\mathrm{E} X} \mathrm{X}$ style emulateapj v. 08/22/09

\title{
PARAMETER ESTIMATION AND CONFIDENCE REGIONS IN THE METHOD OF LIGHT CURVE SIMULATIONS FOR THE ANALYSIS OF POWER DENSITY SPECTRA
}

\author{
Martin Mueller $^{1}$ And Greg Madejski ${ }^{1}$ \\ Draft version November 17, 2021
}

\begin{abstract}
The Method of Light Curve Simulations is a tool that has been applied to X-ray monitoring observations of Active Galactic Nuclei (AGN) for the characterization of the Power Density Spectrum (PDS) of temporal variability and measurement of associated break frequencies (which appear to be an important diagnostic for the mass of the black hole in these systems as well as their accretion state). It relies on a model for the PDS that is fit to the observed data. The determination of confidence regions on the fitted model parameters is of particular importance, and we show how the Neyman construction based on distributions of estimates may be implemented in the context of light curve simulations. We believe that this procedure offers advantages over the method used in earlier reports on PDS model fits, not least with respect to the correspondence between the size of the confidence region and the precision with which the data constrain the values of the model parameters. We plan to apply the new procedure to existing RXTE and XMM observations of Seyfert I galaxies as well as $R X T E$ observations of the Seyfert II galaxy NGC 4945.
\end{abstract}

Subject headings: methods: data analysis — methods: statistical — X-rays: galaxies

\section{INTRODUCTION}

The study of the temporal variability of the X-ray flux from accreting black holes has revealed a complex behavior of the accretion flow (e.g. Mushotzky, Done \& Pounds 1993; Remillard \& McClintock 2006). One widely applied tool for characterizing the variability is the Power Density Spectrum (PDS). The shape of the broad-band PDS as well as the location of identifiable features such as breaks and quasi-periodic oscillations provide the observational constraints for physical models of the system that generates the variability. Of particular interest is the apparent linear scaling between the high-frequency break timescale and the black hole mass in these systems over many orders of magnitude (McHardy et al. 2004).

The analysis of the PDS of Active Galactic Nuclei $(\mathrm{AGN})$ is complicated by the question of how to assign uncertainties to the Fourier amplitudes measured from one observed realization of what is presumed to be a stochastic process (Lawrence et al. 1987; McHardy \& Czerny 1987). A measure of the expected spread in the observed values is essential for the correct interpretation of the Fourier spectrum. In addition, intrinsic properties of the Fourier transform (red noise leak and aliasing), uneven sampling of the time series, and measurement uncertainty in the count rate distort the spectrum; these effects need to be corrected for when quantifying the shape of the broad-band spectrum. A method based on Monte Carlo simulations to determine a reliable measure of the PDS uncertainties and to account for these distortions was first proposed by Done et al. (1992). The main feature of the method is the concept of simulating the possible range of realizations of the underlying process and incorporating the shape-distorting

${ }^{1}$ Kavli Institute for Particle Astrophysics and Cosmology, SLAC National Accelerator Laboratory, 2575 Sand Hill Road, Menlo Park, CA 94025, USA effects of uneven sampling, red noise leak, and aliasing. Uncertainties on the Fourier spectrum are determined using light curves generated from a model for the PDS, and the level of agreement between the model and the data is quantified by a $\chi^{2}$ fit statistic. By analogy to Xray spectral fitting, the application of these observational effects to the chosen model results in the "folded model", which is then used in the comparison to the observations.

Subsequent work by Uttley, McHardy \& Papadakis (2002) (hereafter UMP02), incorporating the recommendations for the simulation of stochastic processes in Timmer \& Koenig (1995), led to a canonical method for the analysis of AGN X-ray light curves, obtained mainly from RXTE and XMM-Newton. The process to be modeled is expressed in the form of a parametric expression for the PDS; depending on the complexity of the model, a varying number of adjustable parameters determine the shape and normalization of the model PDS. In addition to the updated Monte Carlo simulations, the authors present detailed procedures for the statistical evaluation of the model fit, i.e. the assignment of a goodness-of-fit measure and the derivation of confidence regions on model parameters. The fit statistic, which was dubbed the "rejection probability" by subsequent authors, is now different from a standard $\chi^{2}$ statistic. This development toward a statistically more sophisticated technique was influenced by considerations about the resolution of the PDS, which often needs to be compromised to satisfy the conditions under which the $\chi^{2}$ statistic may be used safely (Papadakis \& Lawrence 1993). This new method has found widespread applicability; the results reported in Markowitz et al. (2003) (hereafter M03), McHardy et al. (2004), Markowitz (2005), McHardy et al. (2005), Uttley \& McHardy (2005), McHardy et al. (2007), Summons et al. (2007), Arévalo, McHardy \& Summons (2008), and Marshall. Ryle \& Miller (2008) are all based on it. Our initial report on the PDS of NGC 4945 (Mueller et al. 
2004) similarly took the published method and introduced some additional changes. (In contrast to the above papers, Green, McHardy \& Done (1999), Vaughan, Fabian \& Nandra (2003), Vaughan \& Fabian (2003), and Awaki et al. (2005) implement Monte Carlo simulations for the derivation of uncertainties on the PDS, but use the standard $\chi^{2}$ statistic for the model fit.)

In the general case of fitting a model to a set of data, the derivation of best fit values of the model parameters (called "point estimation" in statistics) involves the identification of the location in parameter space at which the fit statistic attains an extremum ${ }^{2}$. These best fit values are called "estimates"; the recipe for finding an estimate for a particular parameter is called the "estimator". Point estimates by themselves are of limited use. Instead, confidence regions on the fitted model parameters characterize how well the data constrain the model, and goodness-of-fit tests may be applied to test whether the chosen model is an adequate description of the data and whether certain models are favored over others.

The definition of a confidence region is crucial to its proper interpretation. A confidence region (with associated confidence level $C$ ) is a region in parameter space computed from the measured data that has a probability $C$ of containing the true set of parameter values. In other words, if the measurement were repeated, a different confidence region would be obtained for each data set, but a fraction $C$ of them would enclose the (unknown) point in parameter space on which the measured data are based. This of course assumes that the model under consideration is the correct one; if a goodness-of-fit test indicates that the chosen model is a bad description for the data, then confidence regions on its parameters are of little value.

Confidence regions are often interpreted as expressing the precision with which the model parameters may be determined given the data. In practice, for any given fitting procedure, there are usually several plausible methods that produce regions with the required property to make them confidence regions. A useful additional consideration is therefore whether the size of the region that a chosen method returns depends appropriately on the measurement uncertainties. Furthermore, the value of the fit statistic at the location of the best fit should have no or only a weak influence on the size. ${ }^{3}$

We review some of the concepts of model fitting using the $\chi^{2}$ statistic in Section 2, and we show how, in a simple toy model set-up, the $\Delta \chi^{2}$ prescription for finding confidence regions satisfies the consideration above. The "rejection probability" as a fit statistic is evaluated on

2 The use of Monte Carlo simulations for the derivation of the folded model in the case of PDS fitting results in a fit statistic that can not be expressed in closed form as a function of the parameters. Numerical methods therefore need to be employed to search for the location of the extremum.

3 By way of example, in a standard $\chi^{2}$ fit, under the assumption that the chosen model is the correct one and that there are no systematic errors in the measurement, the minimum value of $\chi^{2}$ in a given fit is fully determined by the ratio of the actual amount of statistical fluctuations in the data to the expected amount and does therefore not depend on the size of the measurement uncertainties. In situations encountered in practice, the conditions under which this is true are often violated to a certain degree, such that a small influence on the size of the confidence region cannot be ruled out. the same criteria in Section 3, and the strong dependence of the size of the confidence region on the minimum rejection probability demonstrated. We then introduce the Neyman construction based on simulated distributions of estimates in Section 4 as an alternative to the use of the rejection probability. This paper does not present any actual results obtained from the proposed method. However, we outline in Section 5 possible changes that may occur if PDS model fits obtained using contours of constant rejection probability, including our own work on NGC 4945, were re-examined using the Neyman construction. Section 6 summarizes the paper.

\section{POINT ESTIMATION AND CONFIDENCE REGIONS USING THE $\chi^{2}$ STATISTIC}

The most familiar fit statistic in Astrophysics is without a doubt the $\chi^{2}$ statistic. It applies well to problems where the measured quantities are Gaussian distributed with known uncertainties. Even in cases where that condition is not satisfied, the $\chi^{2}$ statistic can sometimes still yield useful parameter estimates. However, its main attraction lies in the ease with which confidence regions on fitted parameters can be derived if the distributions are Gaussians, namely through the concept of $\Delta \chi^{2}$ (see e.g. Lampton, Margon \& Bowver 1976; Press et al. 1992; Bevington \& Robinson 2003). Any desired significance level $0<\alpha<1$ maps onto a value of $\Delta \chi^{2}$ such that, after determining the best fit values of the model parameters by minimizing $\chi^{2}$, the region in parameter space bounded by the surface of constant $\Delta \chi^{2}$ contains the true set of parameter values with a confidence $C=1-\alpha$.

Let us illustrate the $\Delta \chi^{2}$ procedure on a toy model setup to introduce additional notation that we will refer back to in subsequent sections.

Let $y$ be a physical variable that is expected to be proportional to a single independent variable $x$. As part of an experiment, $y$ is measured for a fixed set of nonequal $x_{i}(i=1, \ldots, N)$. The measurement is expected to result in Gaussian uncertainties on $y$, with a constant standard deviation $\sigma$ independent of $i$. Let $\left\{y_{i}\right\}(i=$ $1, \ldots, N)$ be the set of measurements at the corresponding values $x_{i}$.

We now wish to fit these data with a model $y=k x$. The $\chi^{2}$ fit statistic is then a function of the one model parameter $k$ and the set of observed values $\left\{y_{i}\right\}$ (all sums are over i from 1 to $\mathrm{N})$ :

$$
\chi^{2}\left(k,\left\{y_{i}\right\}\right)=\sum \frac{\left(y_{i}-k x_{i}\right)^{2}}{\sigma^{2}} .
$$

Minimizing $\chi^{2}\left(k,\left\{y_{i}\right\}\right)$ with respect to $k$ yields the estimate $\hat{k}\left(\left\{y_{i}\right\}\right)$ and the minimum value of the fit statistic $\chi_{\min }^{2}\left(\left\{y_{i}\right\}\right)$ :

$$
\hat{k}\left(\left\{y_{i}\right\}\right)=\frac{\sum x_{i} y_{i}}{\sum x_{i}^{2}}
$$

and

$$
\chi_{\min }^{2}\left(\left\{y_{i}\right\}\right)=\sum \frac{\left(y_{i}-\hat{k} x_{i}\right)^{2}}{\sigma^{2}} .
$$

Using these two equations, the expression for the change in $\chi^{2}$ as $k$ is varied evaluates to 


$$
\begin{aligned}
\Delta \chi^{2}\left(k,\left\{y_{i}\right\}\right) & \equiv \chi^{2}\left(k,\left\{y_{i}\right\}\right)-\chi_{\min }^{2}\left(\left\{y_{i}\right\}\right) \\
& =\frac{\sum x_{i}^{2}}{\sigma^{2}}\left(k-\hat{k}\left(\left\{y_{i}\right\}\right)\right)^{2} .
\end{aligned}
$$

To derive the $68 \%$ confidence region (i.e. the " $1 \sigma$ " uncertainties) $)^{4}$ around $\hat{k}\left(\left\{y_{i}\right\}\right)$ (significance $\alpha=0.32$ ), we set $\Delta \chi^{2}\left(k,\left\{y_{i}\right\}\right)=1.00$. The resulting region satisfies

$$
\left|k-\hat{k}\left(\left\{y_{i}\right\}\right)\right|<\frac{\sigma}{\sqrt{\sum x_{i}^{2}}} .
$$

Since we assumed that the measured $y_{i}$ are in fact welldescribed by the model, then each $y_{i}$ has to be drawn from a Gaussian distribution around the true value, i.e.

$$
y_{i} \sim g\left(k_{\text {true }} x_{i}, \sigma\right),
$$

where $g(a, b)$ is a Gaussian distribution with average $a$ and standard deviation $b$, and $\sim$ denotes "drawn from." The estimate $\hat{k}\left(\left\{y_{i}\right\}\right)$ is then drawn from the following probability density function:

$$
\hat{k}\left(\left\{y_{i}\right\}\right) \sim g\left(k_{\text {true }}, \frac{\sigma}{\sqrt{\sum x_{i}^{2}}}\right) .
$$

In other words, if the act of measuring the set $\left\{y_{i}\right\}$ is repeated many times, $\hat{k}\left(\left\{y_{i}\right\}\right)$ will differ from $k_{\text {true }}$ by less than $\sigma / \sqrt{\sum x_{i}^{2}} 68 \%$ of the time. It should now be immediately obvious that the size of the confidence region (Equation 5) is such that in precisely those $68 \%$ of cases the confidence region includes $k_{\text {true }}$, confirming what the confidence region was designed to express about the experiment.

We have thus confirmed that in this simple setup, the $\Delta \chi^{2}$ prescription produces intervals for the model parameter $k$ that satisfy the requirements of confidence intervals. Furthermore, it can be seen from Equation 5] that the size of the confidence interval is proportional to the measurement uncertainty $\sigma$ and independent of $\chi_{\min }^{2}$. We defer to existing publications (specifically Lampton, Margon \& Bowyer 1976) for the extension of these results to higher-dimensional parameter spaces. We only wish to note here that the independence of the size of the confidence region on $\chi_{\text {min }}^{2}$ is guaranteed through the independence of the distribution of $\Delta \chi^{2}$ on $\chi_{\min }^{2}$, as demonstrated in Lampton, Margon \& Bowyer (1976, Appendix IV).

\section{THE REJECTION PROBABILITY}

The measurement of the level of agreement between the model and the observed data in the method of light curve simulations in UMP02 relies on a statistic called by subsequent authors the "rejection probability." It is defined analogous to a $p$-value, with the rejection probability being one minus the $p$-value of the measured $\chi_{\text {dist }}^{2}$ fit statistic. Differences in best-fit rejection probability

4 The term " $1 \sigma$ " is sometimes used to indicate the $68 \%$ confidence region even if the fit statistic is not $\chi^{2}$. In such cases, the standard deviation of the distribution of the parameter may not have the same interpretation, but the confidence regions parameterized by the confidence $C$ are always well-defined. between different models are used to favor one model over the others (e.g. a broken power law model compared to an unbroken power law model), and regions in parameter space where the rejection probability is less than a certain value (e.g. 90\%) are then taken as the confidence regions for the fitted model parameters.

\subsection{Confidence Regions from Rejection Probability}

By analogy to $\chi^{2}$ fitting, the UMP02 procedure for determining confidence regions is equivalent to identifying the region in parameter space where $\chi^{2}$ is less than some critical value. For the $c \%$ confidence region, this critical value is simply the $c$-th percentile of the $\chi^{2}$ distribution with the appropriate number of degrees of freedom and is thus independent of the minimum value of $\chi^{2}$ obtained in the fit. The reason why the authors do not rely on percentiles of $\chi^{2}$ distributions for the determination of confidence regions is that the effective number of degrees of freedom varies with position in parameter space. Deciding on the basis of $p$-values whether a certain point in parameter space is included in the confidence region is therefore more robust.

The region produced in this manner do have the required property to make them confidence regions, i.e., they include the true value of the parameters with the desired probability. However, their sizes depend strongly on the value of the fit statistic at the location of the best fit. If the minimum rejection probability in a fit is just below $90 \%$, the contours of $90 \%$ rejection probability will be found fairly close around the best fit, leading to the erroneous conclusion that the data lend themselves to the placement of very precise limits on the model parameters. Note that a minimum rejection probability of $90 \%$ does not by itself indicate a bad fit, since there is still a $10 \%$ chance of obtaining a fit as bad or worse due simply to statistical fluctuations; we are therefore justified in searching for the confidence region associated with the parameters of such a fit. Conversely, if the minimum rejection probability is very low, the $90 \%$ contours will enclose a large area. Furthermore, if the minimum rejection probability is above $90 \%$, there will be no $90 \%$ confidence region at all.

We illustrate the inverse correlation between the minimum rejection probability and the size of the resulting confidence region schematically in Figure 1. This behavior is apparent in some of the published results (UMP02, M03, McHardy et al. 2007, Summons et al. 2007).

\subsection{The Empirical Distribution of $\chi_{\mathrm{dist}}^{2}$}

The calculation of the rejection probability relies on an approximate determination of the empirical distribution of $\chi_{\text {dist }}^{2}$ : Since the effective number of degrees of freedom is a function of the model parameters, the distribution is rightfully calculated separately for each grid point in parameter space. However, the $\chi_{\text {dist }}^{2}$ values for the simulated Fourier spectra are only calculated at their original grid point and are not the best fit values found by performing the point estimation on each simulated spectrum (see e.g. Press et al. 1992, Section 15.6).

The approximation was most likely introduced due to considerations about computing time, because the minimization of $\chi_{\text {dist }}^{2}$ over the parameter space for the hundreds of simulated spectra that are typically involved incurs a significant computational load. However, given the 


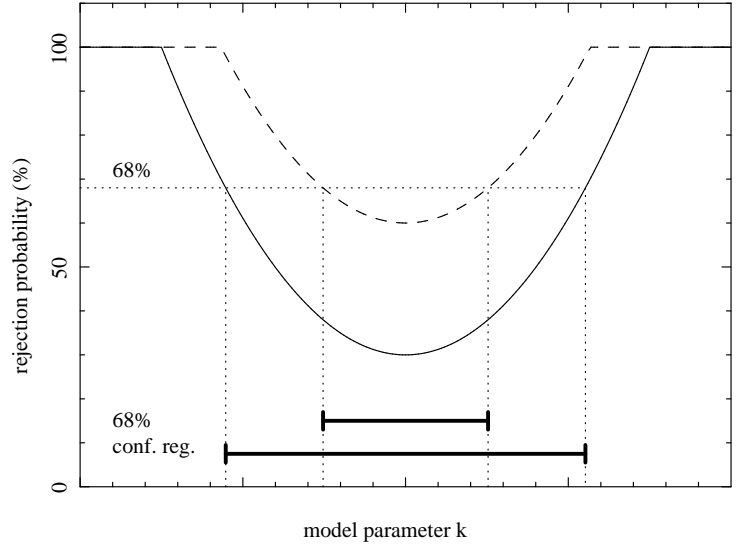

FIG. 1.- Schematic plot illustrating the dependence of the size of the confidence region on the minimum value of the rejection probability. The solid and dashed lines are stylized representations of the behavior of the rejection probability as a function of a model parameter $k$ for fits to two different data sets. Both fits return the same estimate for $k$, but due to statistical fluctuations, the fit indicated by the solid line results in a significantly lower minimum rejection probability. The procedure for determining the $68 \%$ confidence interval on $k$ for each fit is indicated by the dotted lines, showing the projection of the intersection points between the line of $68 \%$ rejection probability and the respective parabola onto the $k$ axis. Even though this is only a schematic representation, and the detailed behavior of the rejection probability as a function of any of the parameters in a real fit may be more complicated, the negative correlation between the size of the confidence region and the minimum value of the rejection probability is expected in general.

advances in computer power since the original method was introduced, the reason for the approximation may well have fallen away.

\section{CONFIDENCE REGIONS AND GOODNESS-OF-FIT TEST} USING SIMULATED DISTRIBUTIONS OF ESTIMATES

We propose the following set of procedures, most importantly the Neyman construction based on distributions of estimates for the derivation of confidence regions, as an alternative to the use of the rejection probability for PDS model fits. The new method returns confidence intervals whose size has the desired property of being independent of the value of the fit statistic at the location of the best fit. Furthermore, it deals very naturally with biased estimators ${ }^{5}$.

Throughout this section, it is assumed that the $\chi_{\text {dist }}^{2}$ fit statistic can be calculated for an arbitrary point in parameter space through the use of simulated light curves. The procedures are however not specific to the $\chi_{\text {dist }}^{2}$ fit statistic; any other statistic which attains an extremum at the location of the best fit may be substituted for $\chi_{\text {dist }}^{2}$.

\subsection{Point Estimation}

$\chi_{\text {dist }}^{2}$ may be used directly for point estimation, i.e. the estimates $\hat{\Theta}_{\text {obs }}$ for the parameters of the model used to describe the observed Fourier spectrum $P_{\text {obs }}(\nu)$ are the values of the parameters at the grid point that minimize $\chi_{\text {dist }}^{2}$. The estimates $\hat{\Theta}_{\text {sim }}$ for any of the simulated light

\footnotetext{
5 Biased estimators are estimators with an expectation value different from the true one. The $\hat{k}$ estimator used in Section 2 is unbiased because its expectation value is $k_{\text {true }}$ (Equation 7). In more complicated situations, such as the PDS fits under consideration here, one does not generally know a priori whether the chosen estimators are biased or not.
}

curves (used further below) can be found similarly by substituting the simulated spectrum in place of the observed spectrum and minimizing $\chi_{\text {dist }}^{2}$ over the parameter space.

\subsection{Goodness-of-Fit and Hypothesis Testing}

In order to test whether the minimum $\chi_{\text {dist }}^{2}$ value of the observed Fourier spectrum signifies an acceptable fit, we use the simulations to determine the distribution from which $\chi_{\text {dist }}^{2}$ is drawn. The null hypothesis is that the measured Fourier spectrum was in fact produced by the model under consideration. Let $\Theta_{\text {best }}$ be our best guess for the true values of the parameters, i.e. the grid point closest to the center of the confidence region. (If the estimators are unbiased, $\Theta_{\text {best }}$ can be set equal to $\hat{\Theta}$.) For each of the simulated light curves generated for $\Theta_{\text {best }}$, we record its best fit $\chi_{\text {dist }}^{2}$ (already found above in the determination of the confidence region). The goodness-of-fit measure is then the familiar $p$-value of the observed spectrum's minimum $\chi_{\text {dist }}^{2}$ compared against this distribution of simulated $\chi_{\text {dist }}^{2}$ values. As such, it expresses the probability that a $\chi_{\text {dist }}^{2}$ value at least as high as the measured one would be obtained by chance; a $p$-value smaller than the desired significance level (e.g. $5 \%$ ) indicates that the null hypothesis can be rejected.

The reason for choosing $\Theta_{\text {best }}$ over any other grid point is that the distribution of the minimum values of $\chi_{\text {dist }}^{2}$ may depend on $\Theta$. In standard $\chi^{2}$ fitting, the distribution of $\chi_{\min }^{2}$ is independent of $\Theta$, being in fact the $\chi^{2}$ distribution with the appropriate number of degrees of freedom. In the framework of Fourier spectral fits, this independence appears to be broken, such that the effective number of degrees of freedom is a function of the model parameters, plausibly because the degree to which adjacent bins in the Fourier spectrum are correlated depends on the amount of red noise leak (Mueller et al., in preparation). Using $\Theta_{\text {best }}$ ensures that the $\chi_{\text {dist }}^{2}$ distribution thus found approximates as closely as possible the one from which the measured $\chi_{\text {dist }}^{2}$ was in fact drawn.

Note that, up to the approximation to the distribution of $\chi_{\text {dist }}^{2}$ used by UMP02, this procedure is essentially equivalent to the calculation of the rejection probability. The $p$-value is however only used as a goodness-of-fit measure and not for finding the confidence intervals.

If more than one model are under consideration to explain the measured data, e.g. when one would like to test for the presence of a break in the Fourier spectrum, a decision statistic for hypothesis testing needs to be set up. In the framework of the $\chi_{\text {dist }}^{2}$ fit statistic, the difference in best fit $\chi_{\text {dist }}^{2}$ values between two models is a natural choice for such a statistic (by analogy to the F-test for the $\chi^{2}$ fit statistic). The simulations can once again be used to determine the distribution of this difference, from which the critical value corresponding to a desired power of the test ("statistical significance") may be derived. We do not further elaborate on this procedure here, since the numbers and decisions involved depend on a balance between the sensitivity and specificity of the test that can only be calculated using actual simulations.

\subsection{Confidence Regions}

We implement the Neyman construction (Neyman 1937) based on simulated distributions of estimates to 

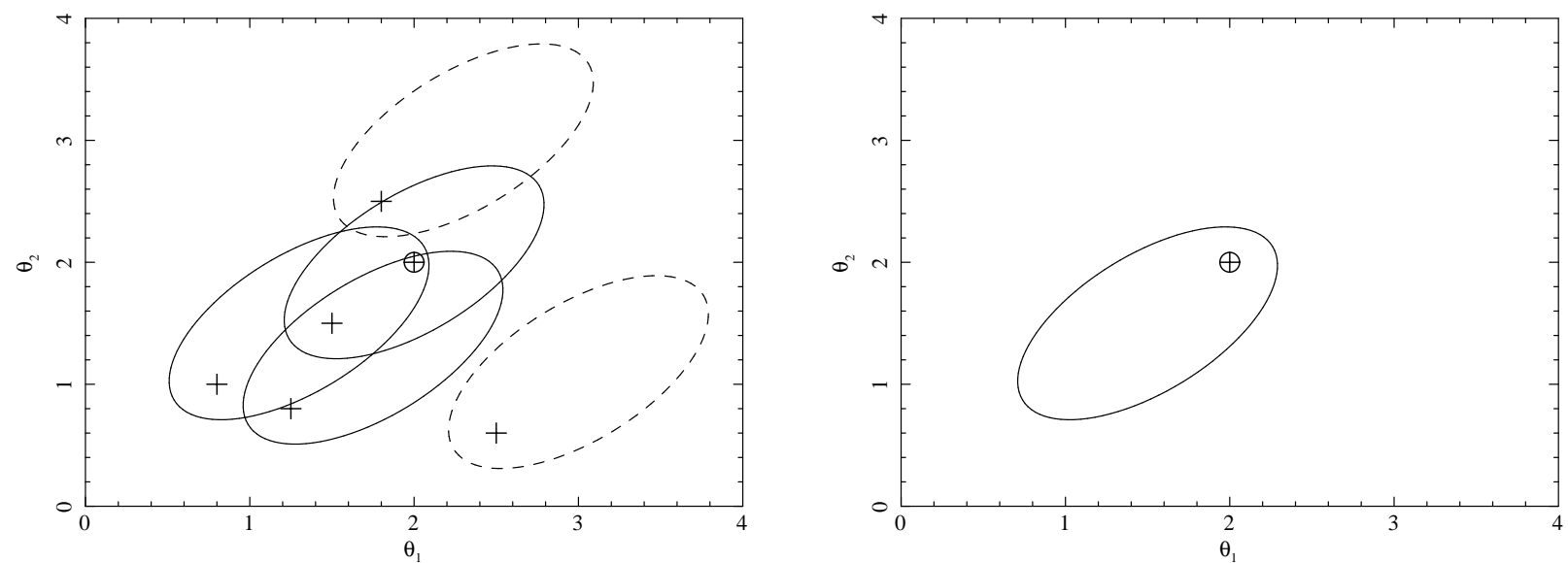

FIG. 2. - Schematic illustration of the Neyman construction applied to a model fit with two adjustable parameters $\theta_{1}$ and $\theta_{2}$. The plot on the left shows elliptical regions obtained from the distribution of the estimates that encompass an unspecified, but constant, fraction $C$ of the distribution. The corresponding trial values of the parameters for each ellipse are indicated by the crosses. The offset between the crosses and the centers of the ellipses implies a bias in the estimators, kept constant as a function of the parameters in this simple example. The location of the observed best-fit $\left(\hat{\theta_{1}}, \hat{\theta_{2}}\right)$ is denoted by the cross-hairs. The solid ellipses include the observed best-fit values of the parameters, the dashed ones do not. By the prescription of the Neyman construction, the parameter values associated with the solid ellipses are added to the confidence region, the others are not. The plot on the right shows the elliptical confidence region $($ confidence $=$ $C$ ) that would be obtained if this procedure were to be repeated for all possible trial values of the parameters. The observed best-fit values are once more indicated by the cross-hairs. Note how the estimator bias identified earlier results in a confidence region whose center is offset from the observed best-fit values.

find confidence limits on model parameters: Let $C$ be the desired confidence, e.g. $68 \%$ or $90 \%$, and $\hat{\Theta}_{\text {obs }}$ the estimates for the observed Fourier spectrum as found above. Consider now an arbitrary grid point in parameter space, $\Theta_{\text {trial }}$. Using the simulated light curves generated for that point, we can determine the distribution of estimates $\hat{\Theta}_{\text {sim }}$ and derive a region in parameter space that encloses a fraction $C$ of them. If $\hat{\Theta}_{\text {obs }}$ is inside that region, $\Theta_{\text {trial }}$ is included in the confidence region, otherwise it is not. Figure 2 shows this graphically in an imagined two-parameter model fit.

It is easy to see that the size of the confidence regions thus obtained is independent of the value of the minimum $\chi_{\text {dist }}^{2}$; no information about the measured data enters the calculation of the distribution of estimates, and only the estimates $\hat{\Theta}_{\text {obs }}$ are used in the subsequent mapping of the confidence region. Also, the distribution of estimates, being a measure of the possible range of parameter values that might be observed given the design of the measurement, will be broad or narrow depending on the uncertainties in the observed Fourier spectrum. Therefore, the size of the confidence region will scale with the uncertainties, preserving the intended interpretation that the size of the confidence region expresses the precision with which fitted model parameters can be determined.

As a consequence of using distributions of estimates in the Neyman construction, biased estimators will be corrected, and the region returned by the algorithm has the required property of enclosing the true values of the parameters with probability $C$. In principle, if any of the estimators are strongly biased, $\hat{\Theta}_{\text {obs }}$ might lie outside the confidence region. The confidence region is however always more meaningful in the determination of probable values of model parameters than a (possibly biased) point estimate.

Note also that the shape of the region enclosing the fraction $C$ of all simulations may be freely chosen by the user. This freedom is intrinsic to the Neyman construc- tion. However, in order to obtain the tightest possible constraints on the parameters, the smallest region should be used.

A complication arises out of the limitation of being able to evaluate $\chi_{\text {dist }}^{2}$ only on a grid in parameter space. The distributions of the estimates are therefore composed of finite-size volume elements centered on each grid point. The use of smoothed approximations to the empirically derived distributions can eliminate this step-wise behavior; for a one-dimensional parameter space, if the distribution of the estimate is sharply peaked, one might use a Gaussian fit and determine from the fitted average and standard deviation whether the trial point lies inside the confidence interval. Similarly, in two dimensions, the use of ellipses fitted to the empirical distribution to enclose the required fraction of simulated estimates might be appropriate.

Let us illustrate the procedure of finding confidence regions using simulated distributions of estimates on the toy model introduced in Section 2. The difference to the procedure described above is that the estimate $\hat{k}$ can be found analytically and does not rely on a grid search using simulations. However, let us suppose that simulations were set up for this simple problem. We use Equation 2 to calculate $\hat{k}_{\text {obs }}$ for the measured data $\left\{y_{i}\right\}$. Let $k_{\text {trial }}$ be an arbitrarily chosen real number, for which we want to determine whether it is inside the confidence interval. Using simulations with $k_{\text {trial }}$ as the "true" parameter value (i.e. randomizations of the observations as given by Equation [6), we would then find (Equation 7) that the probability density function of the estimates for this trial value is given by $g\left(k_{\text {trial }}, \sigma / \sqrt{\sum x_{i}^{2}}\right)$, i.e. a Gaussian distribution centered on $k_{\text {trial }}$. (In this toy model, the distribution of the estimates is translationally invariant under changes in $k_{\text {trial }}$; this feature is not expected in general.) The smallest interval enclosing $68 \%$ of the simulations is comprised of the values within one standard deviation from $k_{\text {trial }}$, and by the prescription of the method $k_{\text {trial }}$ is included in the confidence region if 
and only if

$$
\left|k_{\text {trial }}-\hat{k}_{\text {obs }}\right|<\frac{\sigma}{\sqrt{\sum x_{i}^{2}}},
$$

thus recovering the confidence region in Equation 5 .

\subsubsection{The Confidence Interval for the Model Normalization}

The model to be fit to the data usually includes an overall normalization factor that carries through to the model prediction as a multiplicative factor. In this situation, the derivation of confidence intervals on the model normalization can be simplified. In practice, simulations need only be done once for an arbitrary normalization, since the model prediction and uncertainties for any other normalization may be calculated simply by scaling. (For a discussion of the complications introduced by measurement uncertainties, see Appendix A.1) For point estimation, the best fit normalization at any point in parameter space ${ }^{6}$ can easily be found, either analytically or through a numerical search. This procedure is unchanged from UMP02 (section 4.2). The estimates for the remaining parameters, either for the measured data or for any of the simulated spectra that may be substituted for it, are then once again the values of the parameters at the grid point in the remaining parameter space where $\chi_{\text {dist }}^{2}$ attains a minimum.

For the derivation of confidence regions, we again wish to determine whether a certain grid point in parameter space, with normalization $N_{\text {trial }}$ and remaining parameters $\Theta_{\text {trial }}$, is included at a given significance level. Let $N_{0}$ be the original normalization with which the light curves at $\Theta_{\text {trial }}$ were simulated, and $f_{0}(\hat{N}, \hat{\Theta})$ the corresponding probability density function of the estimate distribution with its dependence on the normalization estimate $\hat{N}$ and the estimates of the remaining model parameters $\hat{\Theta}$. Because of the multiplicative nature of the model normalization, this distribution becomes scaleinvariant along the $\hat{N}$ axis (see Appendix $(\mathrm{A}$ ), such that if a different normalization had been used to simulate those light curves, the distribution would simply be an appropriately scaled version of $f_{0}(\hat{N}, \hat{\Theta})$.

The point estimation on the observed Fourier spectrum defines the location of the best fit, given by $\left(\hat{N}_{\text {obs }}, \hat{\Theta}_{\text {obs }}\right)$. We now determine the region $R$ in the $(\hat{N}, \hat{\Theta})$ space that encloses the required fraction $C$ of the estimate distribution (e.g. 68\%). Now consider the line in parameter space along which $\hat{\Theta}=\hat{\Theta}_{\text {obs }}$, i.e. the line parallel to the $\hat{N}$ axis that passes through the best fit. This line either intersects the boundary of $R$ in a finite number of points, or else no intersection points exist. The region $R$ is in most cases convex, such that there are either zero or two intersection points. We will only consider these two cases here; the procedure is easily generalized to nonconvex regions that may result in additional intersection points.

In the case of zero intersection points, $\left(N_{\text {trial }}, \Theta_{\text {trial }}\right)$ is excluded from the confidence region for all values of $N_{\text {trial }}$. In the other case, let us denote the values of the

6 The parameter space now under consideration excludes the model normalization as a parameter, due to its special treatment. normalization at the two intersection points by $\hat{N}_{0 \text {,low }}$ and $\hat{N}_{0 \text {,high. }}$ Because of the scale-invariance of the estimate distribution, these values are proportional to the original normalization $N_{0}$, such that, for any other normalization $N$ that could have been used to generate the light curves,

$$
\hat{N}_{\text {low }}(N)=\frac{N}{N_{0}} \hat{N}_{0, \text { low }}
$$

and

$$
\hat{N}_{\text {high }}(N)=\frac{N}{N_{0}} \hat{N}_{0, \text { high }}
$$

The condition for $\left(N_{\text {trial }}, \Theta_{\text {trial }}\right)$ to be included in the confidence region now reduces to whether the observed value of the normalization is located between these two bounds, i.e.

$$
\hat{N}_{\text {low }}\left(N_{\text {trial }}\right) \leq \hat{N}_{\text {obs }} \leq \hat{N}_{\text {high }}\left(N_{\text {trial }}\right),
$$

which is equivalent to the condition on $N_{\text {trial }}$

$$
N_{0} \frac{\hat{N}_{\text {obs }}}{\hat{N}_{0, \text { high }}} \leq N_{\text {trial }} \leq N_{0} \frac{\hat{N}_{\text {obs }}}{\hat{N}_{0, \text { low }}} .
$$

In summary, the estimate distribution found from light curves simulated at $\Theta_{\text {trial }}$, with normalization $N_{0}$, may be used to derive the bounds $\hat{N}_{0 \text {,low }}$ and $\hat{N}_{0 \text {, high }}$, from which the limits on $N_{\text {trial }}$ (for a given $\Theta_{\text {trial }}$ ) may be calculated. The confidence region in the full $(N, \Theta)$ parameter space finally may be mapped out by repeating the procedure for different values of $\Theta_{\text {trial }}$.

\section{DISCUSSION}

Applying the criterion whether the procedure for determining confidence regions returns regions whose size scales appropriately with the uncertainties in the data, we believe that the Neyman construction based on simulated distributions of estimates offers a viable and advantageous alternative to the use of the rejection probability. While neither UMP02 nor the authors of the subsequent papers utilizing the method make specific claims regarding the statistical properties of the regions obtained, anyone not familiar with the data analysis at a sufficient level of detail will tend to interpret the quoted uncertainties on the best-fit values of the model parameters as indicative of the precision with which the data constrain those values.

We wish to stress however that this does not imply that previously reported results are inherently flawed. The confidence limits on break frequencies and power law indices for AGN PDS fits may turn out to be different under the application of the new method, but it remains to be seen whether any of these changes are large enough to substantially change the interpretation of the observations. Specifically, we do not expect that the linear scaling between break timescale and black hole mass (McHardy et al. 2004) will be affected even if the confidence intervals on some of the data points were to be modified.

It is likely that the precision with which the break frequencies for Fairall 9, NCG 4151 (both from M03), and 
MCG-6-30-15 (UMP02) have been reported is too optimistic. Similarly, the confidence regions for the peak frequencies of the Lorentzians in the fit to the PDS of Ark 564 may be too small, especially considering that even a small increase in the size of the confidence contour in a plot where the axes are the logarithms of the peak frequencies (Figure 9 in their paper) has a disproportionate effect on the uncertainties on the frequencies themselves. On the other hand, some break frequencies may in fact be better determined with current data than reported in the literature. Examples of fits where the minimum rejection probability turned out to be particularly low include NGC 5548 and NGC 3516 (M03).

A related issue is the use of contours of constant rejection probability as confidence limits on combinations of parameters, such as the ratio of break frequencies (Figure 11 in McHardy et al. 2005 and Figure 6 in Uttley \& McHardy 2005). Limits on this ratio are used as key pieces of evidence to motivate the association of the AGNs under consideration (NGC 3227 and MCG-6$30-15$ ) with the analogue of the high/soft accretion state in galactic black hole X-ray binaries. Given that the confidence regions were derived using the rejection probability, the quoted confidence values with which certain ranges of ratios are excluded in those reports may or may not in fact be supported by the data. We do not expect that the use of the new method will alter the general direction of these results, i.e. that the ratio of break frequencies in these AGN is likely to be higher than expected for the low/hard state, but a re-analysis of the observations focusing on the doubly-broken power law model might be warranted. The calculation of the statistical significance with which the model where the ratio of these break frequencies is fixed at a value of 30 may be rejected in favor of the original model where both break frequencies are allowed to vary forms an additional test on these data. If both of these lines of evidence produce mutually consistent results, the case for the classification of these AGN as analogues of galactic X-ray binaries in the high/soft state will be strengthened.

On the question of the statistical significance of breaks in the PDS of AGN, we believe that additional work is needed to make the values that have been reported more secure. Table 5 in M03 lists the quantity $\Delta \sigma$ that was designed to express the increase in likelihood of the fit once a break is added to the PDS model. It is however not clear from the description whether $\Delta \sigma$ was calculated using the rejection probability or the underlying $\chi_{\text {dist }}^{2}$ values. As outlined in Section 4.2, the $\chi_{\text {dist }}^{2}$ fit statistic does lend itself to the formulation of such a hypothesis test. A validation of critical values of differences in $\chi_{\text {dist }}^{2}$ and their corresponding statistical significances will be required. This includes using the simulated light curves to calculate type I and type II errors (rate of false positives and false negatives) or, equivalently, the specificity and sensitivity of the hypothesis test. As far as we are aware, the amount and quality of data needed to effect a reliable detection of a break at a significance level of $5 \%$, say, is an unanswered question. A systematic investigation in this area, using both real and simulated data, might uncover general considerations that would be invaluable for the design of future observatories for AGN timing research.
The Monte Carlo method for calculating folded models to include observational effects may have applications outside of PDS fits to AGN X-ray light curves. In particular, the associated procedure of finding confidence limits on fitted parameters using simulated estimate distribution could be used for X-ray or $\gamma$-ray spectral fits in the low counts-per-bin limit, where the discrete nature of the Poisson process becomes important. The study of the PDS of galactic X-ray binaries may benefit from an application of the Monte Carlo method as well. The shorter time scales for characteristic variations and the extensive archive of X-ray observations allow for a much more detailed investigation into the shape of the broad-band variability spectrum, including the direct observation of independent realizations of the underlying process (e.g. Pottschmidt et al. 2003; Done \& Gierlinski 2005). The measurement of the distribution of power in individual frequency bins is of particular interest in this case. Competing physical models for the variability in these sources predict distinctive properties of the stationarity and degree of stochasticity of the process underlying the observations (e.g. Poutanen \& Fabian 1999; Maccarone \& Coppi 2002; Minutti \& Fabian 2004; Uttley. McHardy \& Vaughan 2005; Zycki \& Maciolek-Niedzwiecki 2005). Adopting the Monte Carlo simulations for the analysis of galactic X-ray binaries, specifically the comparison between predicted and observed distributions of the Fourier amplitude, may lead to tests of certain elements of these models. Furthermore, tools beyond the PDS for the investigation of these kinds of stochastic processes, such as the bispectrum (Vaughan \& Uttley 2007), are more sophisticated in their treatment of the underlying variability process, but they will also continue to, at least for a while, produce results that are not as validated in their interpretation as those derived from standard Fourier analysis. Monte Carlo simulations will likely remain essential for the important comparison of these tools to the PDS; a solid statistical foundation is in turn essential for these simulations.

\section{CONCLUSION}

Evaluated by the criterion whether the sizes of the confidence regions express the precision with which the data constrain the model parameters, we have shown that the use of simulated distributions of estimates (Section 4.3) is preferable to the rejection probability (Section 3.1). Confidence regions determined from the latter do have the required property of enclosing the true values of the parameters with the given probability, but their size is highly variable depending on the minimum value of the fit statistic at the location of the best fit. The method based in the former is computationally more intensive, but is the only way known to us to derive meaningful uncertainties on fitted model parameters in the absence of a better-understood fit statistic.

The end products of the application of the set of procedures in Section 4.1 through 4.3 to the observations of an AGN are the best fit values of the parameters for the model-dependent description of the PDS, the associated confidence limits, and the goodness-of-fit of the model ( $p$ value of the minimum $\chi_{\text {dist }}^{2}$ ). Depending on the nature of the investigation, more sophisticated statistical tests may be employed to test different hypotheses against the 
same data set, or to quantify the observed variations in the parameter values between different AGN.

We are in the process of applying the new method to the $R X T E$ observation of the Seyfert II galaxy NGC 4945 for which we reported first results in Mueller et al. (2004) and plan to re-analyze the existing archival $R X T E$ and $X M M-N e w t o n$ observations of Seyfert I galaxies with the updated procedure. While we don't expect the conclusions drawn from the analysis of these observations to change significantly, this will put the investigation into the shape of the PDS in AGN on a statistically more solid foundation and make the interpretation of the results easier.

\section{ACKNOWLEDGMENTS}

We are indebted to the anonymous referee for much valued input on an earlier version of this publication, especially concerning the development of the analysis method based on the rejection probability. We would furthermore like to acknowledge Alex Markowitz for providing the initial impetus for the critical examination of the rejection probability, and Chris Done and Piotr Życki for the ongoing collaboration on the analysis of the NGC 4945 observations. We also thank Jeff Scargle, and especially Frank Porter, for many fruitful discussions on statistical techniques and their expertise on the associated terminology. This research was supported in part by the U.S. Department of Energy Contract DE-AC02$76 \mathrm{SF} 00515$ to the SLAC National Accelerator Laboratory.

\section{APPENDIX}

THE SCALE-INVARIANCE OF THE ESTIMATE DISTRIBUTION FOR THE MODEL NORMALIZATION

The special treatment of the model normalization in the derivation of confidence regions relies on a property of the estimate distribution under the conditions mentioned in the text (Section 4.3.1), namely that the normalization carries through to the model prediction as a multiplicative factor.

Let $N_{0}$ be the normalization (hereafter called the "input normalization") that was used to generate a set of light curves at an arbitrary point in parameter space $\Theta_{\text {trial }}$, and let $\left\{P_{\text {in }}\left(\nu_{i}\right)\right\}$ be the Fourier spectrum of one of them, where $\nu_{i}$ are the frequencies over which the spectrum is measured. Additionally, let $\left(\hat{N}_{\text {in }}, \hat{\Theta}_{\text {in }}\right)$ be the estimates for this light curve that were found as part of the procedure to determine the estimate distribution (see Section 4.3 for details). Because the normalization is an overall multiplicative factor in the generation of these light curves, the $\left\{P_{\text {in }}\left(\nu_{i}\right)\right\}$ values are proportional to $N_{0}$.

The model to be fit to these data can be written as $P(\nu, N, \Theta)=N P_{r}(\nu, \Theta)$, where $N$ is the model normalization and $P_{r}(\nu, \Theta)$ the function describing the dependence of the model on the remaining parameters $\Theta$. The folded model at the point in parameter space given by $N$ and $\Theta$ is summarized in two variables for each frequency bin: the average power $\overline{P_{\operatorname{sim}}}\left(\nu_{i}, N, \Theta\right)$ and the standard deviation $\Delta P_{\operatorname{sim}}\left(\nu_{i}, N, \Theta\right)$ (for details, see Section 4.2 in UMP02). Both of these scale with $N$ :

$$
\overline{P_{\text {sim }}}\left(\nu_{i}, N, \Theta\right)=N \overline{P_{\text {sim }, r}}\left(\nu_{i}, \Theta\right)
$$

and

$$
\Delta P_{\operatorname{sim}}\left(\nu_{i}, N, \Theta\right)=N \Delta P_{\operatorname{sim}, r}\left(\nu_{i}, \Theta\right)
$$

where $\overline{P_{\text {sim }, r}}\left(\nu_{i}, \Theta\right)$ and $\Delta P_{\text {sim }, r}\left(\nu_{i}, \Theta\right)$ constitute the folded model for $N=1$. The fit statistic

$$
\chi_{\text {dist }}^{2}\left(N, \Theta,\left\{P_{\text {in }}\left(\nu_{i}\right)\right\}\right)=\sum_{i}\left(\frac{P_{\text {in }}\left(\nu_{i}\right)-N \overline{P_{\text {sim }, r}}\left(\nu_{i}, \Theta\right)}{N \Delta P_{\text {sim }, r}\left(\nu_{i}, \Theta\right)}\right)^{2}
$$

is invariant under changes in the input normalization $N_{0} \rightarrow \eta N_{0}(\eta>0)$ if the same multiplicative factor is applied to the model normalization $N$. As a consequence, since $\hat{N}_{\text {in }}$ and $\hat{\Theta}_{\text {in }}$ are the estimates for this simulated light curve for $\eta=1$, then $\left(\eta \hat{N}_{\text {in }}\right)$ and $\hat{\Theta}_{\text {in }}$ would have been the estimates if the original normalization had been different by a factor $\eta$. This applies to all simulated spectra; therefore the distribution of the estimates $\left(\hat{N}_{\text {in }}\right.$, $\left.\hat{\Theta}_{\text {in }}\right)$ will be scale-invariant along the $\hat{N}$ axis: Let $f_{0}(\hat{N}, \hat{\Theta})$ be the estimate distribution for the original input normalization $N_{0}$ (i.e. $\left.\eta=1\right)$. For any other value of $\eta$, the estimate distribution is then

$$
f(\hat{N}, \hat{\Theta})=\frac{1}{\eta} f_{0}\left(\frac{\hat{N}}{\eta}, \hat{\Theta}\right) .
$$

Note that the above does not require that the normalization be uncorrelated with the other model parameters. The invariance of the fit statistic is preserved even if such correlations exist.

\section{Influence of Measurement Uncertainties}

In the context of PDS model fits, the measurement uncertainties in the light curve manifest themselves as an additional noise component in the Fourier spectrum (Poisson level). The scaling of the model prediction with the 
normalization factor is only approximate in this case, since the Poisson level is constant and does not scale with the model normalization. However, the intrinsic variability in the light curve by design usually dominates over the Poisson level. The confidence interval on the model normalization derived while ignoring this complication is therefore expected to approximate closely the more correct one that would be obtained through the usual prescription of simulating light curves with different normalizations and deriving the distribution of the estimates in each case.

\section{Applicability to PDS with Logarithmically Averaged Power}

In the canonical method of UMP02, $\overline{P_{\text {sin }}}\left(\nu_{i}\right)$ is actually the average of the logarithm of the periodogram power, which is motivated by the considerations in Papadakis \& Lawrence (1993). The logarithm of the model normalization $N$ therefore enters the model prediction as an additive constant, while the uncertainties $\Delta P_{\operatorname{sim}}\left(\nu_{i}\right)$, being standard deviations on what are now logarithmic power values whose spread is unaffected by the model normalization, are independent of $N$. The estimate distribution is then translationally invariant along the $\log \hat{N}$ axis, and the expression for the bounds on $N_{\text {trial }}$ turns out to be the same as for the linear case (Equation [12).

Note however that different numerical values for these bounds may be obtained depending on whether the estimate distribution is expressed as a function of $\hat{N}$ or $\log \hat{N}$. The shape and extent of the region $R$ encompassing the desired fraction of the estimate distribution may vary; the smallest such region for example will in general be different depending on the choice of variables. In a complete description of the analysis method, it will be important to state which variable was used.

\section{REFERENCES}

Arévalo, P., McHardy, I. M., Summons, D. P. 2008, MNRAS, 388, 211

Awaki, H., Murakami, H., Leighly, K. M., Matsumoto, C., Hayashida, K., Grupe, D. 2005, ApJ, 632, 793

Bevington, P. R., Robinson, D. K. 2003, Data Reduction and Error Analysis for the Physical Sciences, $3^{\text {rd }}$ edition, McGraw-Hill

Done, C., Gierliński, M. 2005, MNRAS, 364, 208

Done, C., Madejski, G. M., Mushotzky, R. F., Turner, T. J., Koyama, K., Kunieda, H. 1992, ApJ, 400, 138

Green, A. R., McHardy, I. M., Done, C. 1999, MNRAS, 305, 309

Lampton, M., Margon, B., Bowyer, S. 1976, ApJ, 208, 177

Lawrence, A., Watson, M. G., Pounds, K. A., Elvis, M. 1987, Nature, 325, 694

Maccarone, T. J., Coppi, P. S. 2002, MNRAS, 336, 817

Markowitz, A. 2005, ApJ, 635, 180

Markowitz, A. et al. 2003, ApJ, 593, 96 (M03)

Marshall, K., Ryle, W. T., Miller, H. R. 2008, ApJ, 677, 880

McHardy, I. M., Arévalo, P., Uttley, P., Papadakis, I. E., Summons, D. P., Brinkmann, W., Page, M. J. 2007, MNRAS, 382,985

McHardy, I., Czerny, B. 1987, Nature, 325, 696

McHardy, I. M., Gunn, K. F., Uttley, P., Goad, M. R. 2005, MNRAS, 359, 1469

McHardy, I. M., Papadakis, I. E., Uttley, P., Page, M. J., Mason, K. O. 2004, MNRAS, 348, 783

Minutti, G., Fabian, A. C. 2004, MNRAS, 349, 1435

Mueller, M., Madejski, G., Done, C., Życki, P. 2007, in Kaaret, P., Lamb, F. K., Swank, J. H., eds., X-Ray Timing 2003: Rossi and Beyond, American Institute of Physics, Melville, NY, 714, 190
Mushotzky, R. F., Done, C., Pounds, K. A. 1993, ARA\&A, 31, 717

Neyman, J. 1937, Philosophical Transactions of the Royal Society of London, Series A, Mathematical and Physical Sciences, 236, 333

Papadakis, I. E., Lawrence. A. 1993, MNRAS, 261, 612

Pottschmidt, K. et al. 2003, A\&A, 407, 1039

Poutanen, J., Fabian, A. C. 1999, MNRAS, 306, L31

Press, W. H., Teukolsky, S. A., Vetterling, W. T., Flannery, B. P. 1992, Numerical Recipes, Cambridge University Press, Cambridge

Remillard, R. A., McClintock, J. E. 2006, ARA\&A, 44, 49

Summons, D. P., Arévalo, P., McHardy, I. M., Uttley, P., Bhaskar, A. 2007, MNRAS, 378, 649

Timmer, J., Koenig, M. 1995, A\&A, 300, 707

Uttley, P., McHardy, I. M. 2005, MNRAS, 363, 586

Uttley, P., McHardy, I., Papadakis, I. 2002, MNRAS, 332, 231 (UMP02)

Uttley, P., McHardy, I. M., Vaughan, S. 2005, MNRAS, 359,345

Vaughan, S., Fabian, A. C. 2003, MNRAS, 341, 496

Vaughan, S., Fabian, A. C., Nandra, K. 2003, MNRAS, 339, 1237

Vaughan, S., Uttley, P. 2007, in Cohen, L. ed., Noise and

Fluctuations in Photonics, Quantum Optics, and

Communications, Proc. SPIE, 6603, 660314

Życki, P., Niedźwiecki, A. 2005, MNRAS, 359, 308 